\title{
DART-bid (Dose-differentiated accelerated radiation therapy, 1.8 Gy twice daily)-a novel approach for non-resected NSCLC: final results of a prospective study, correlating radiation dose to tumor volume
}

Karl Wurstbauer ${ }^{1,3^{*}}$, Heinz Deutschmann ${ }^{1}$, Karin Dagn ${ }^{1}$, Peter Kopp ${ }^{1}$, Franz Zehentmayr ${ }^{1}$, Bernd Lamprecht ${ }^{2}$, Peter Porsch², Birgit Wegleitner ${ }^{2}$, Michael Studnicka² and Felix Sedlmayer ${ }^{1}$

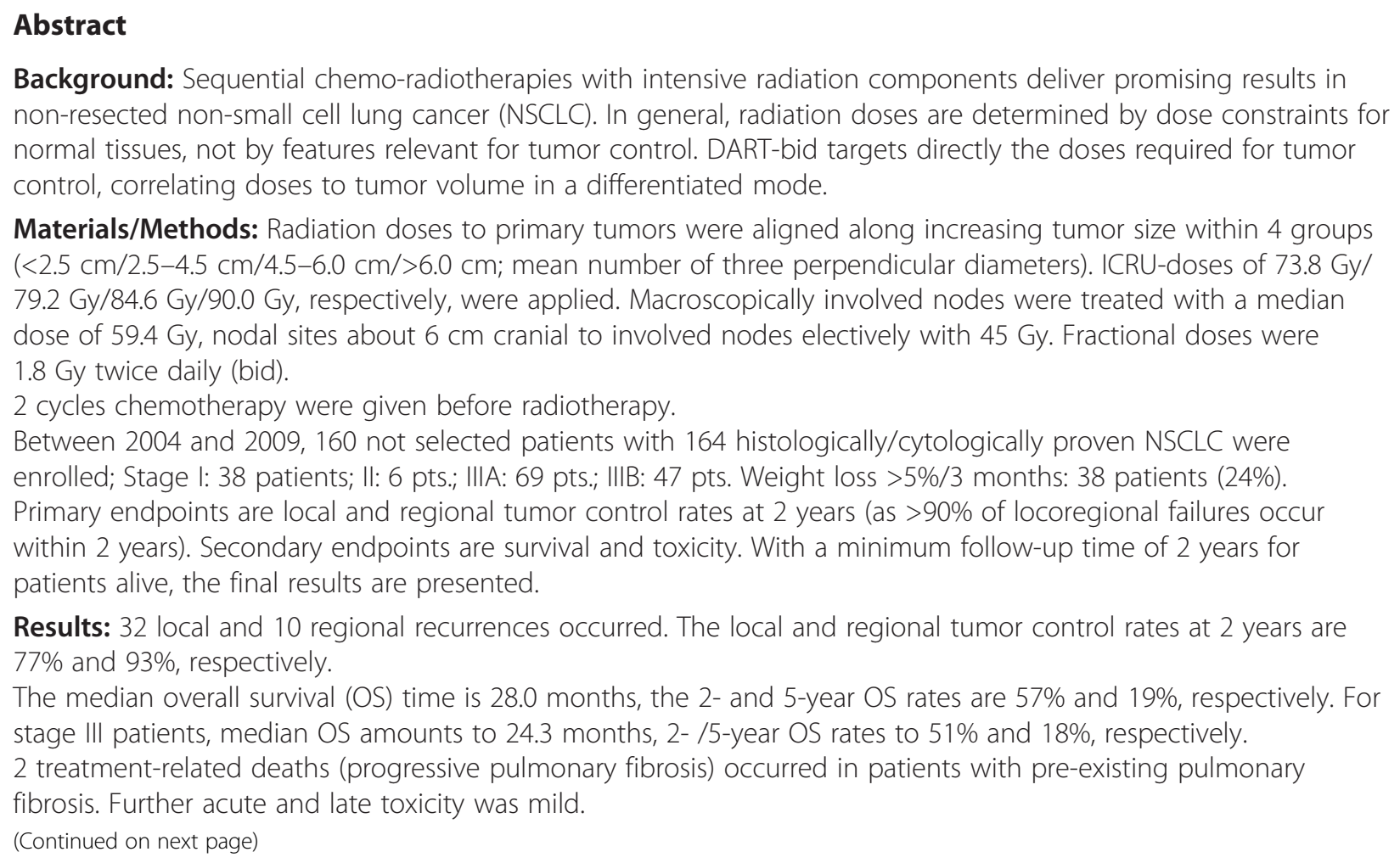

Background: Sequential chemo-radiotherapies with intensive radiation components deliver promising results in non-resected non-small cell lung cancer (NSCLC). In general, radiation doses are determined by dose constraints for normal tissues, not by features relevant for tumor control. DART-bid targets directly the doses required for tumor control, correlating doses to tumor volume in a differentiated mode.

Materials/Methods: Radiation doses to primary tumors were aligned along increasing tumor size within 4 groups ( $<2.5 \mathrm{~cm} / 2.5-4.5 \mathrm{~cm} / 4.5-6.0 \mathrm{~cm} />6.0 \mathrm{~cm}$; mean number of three perpendicular diameters). ICRU-doses of $73.8 \mathrm{~Gy} /$ 79.2 Gy/84.6 Gy/90.0 Gy, respectively, were applied. Macroscopically involved nodes were treated with a median dose of $59.4 \mathrm{~Gy}$, nodal sites about $6 \mathrm{~cm}$ cranial to involved nodes electively with $45 \mathrm{~Gy}$. Fractional doses were 1.8 Gy twice daily (bid).

2 cycles chemotherapy were given before radiotherapy.

Between 2004 and 2009, 160 not selected patients with 164 histologically/cytologically proven NSCLC were enrolled; Stage I: 38 patients; II: 6 pts.; IIIA: 69 pts.; IIIB: 47 pts. Weight loss >5\%/3 months: 38 patients (24\%). Primary endpoints are local and regional tumor control rates at 2 years (as $>90 \%$ of locoregional failures occur within 2 years). Secondary endpoints are survival and toxicity. With a minimum follow-up time of 2 years for patients alive, the final results are presented.

Results: 32 local and 10 regional recurrences occurred. The local and regional tumor control rates at 2 years are $77 \%$ and $93 \%$, respectively.

The median overall survival (OS) time is 28.0 months, the 2 - and 5-year OS rates are 57\% and 19\%, respectively. For stage III patients, median OS amounts to 24.3 months, 2- /5-year OS rates to $51 \%$ and $18 \%$, respectively.

2 treatment-related deaths (progressive pulmonary fibrosis) occurred in patients with pre-existing pulmonary fibrosis. Further acute and late toxicity was mild.

(Continued on next page)

\footnotetext{
* Correspondence: k.wurstbauer@salk.at

'Department of Radiation Oncology and radART-Institute for research and

development on Advanced Radiation Technologies, Paracelsus Medical

University, Salzburg, Austria

${ }^{3}$ Universitätsklinik für Radiotherapie, Müllner Hauptstrasse 48, A-5020

Salzburg, Austria

Full list of author information is available at the end of the article
} 
(Continued from previous page)

Conclusions: This novel approach yields a high level of locoregional tumor control and survival times. In general it is well tolerated. In all outcome parameters it seems to compare favourably with simultaneous chemoradiotherapies, at present considered 'state of the art'; and is additionally amenable for an unselected patient population.

Keywords: Lung cancer, Non-small cell lung cancer, Accelerated radiotherapy, Conformal radiotherapy, Target splitting, Prospective clinical trials, Combined modality, Treatment time, Accelerated repopulation, DART-bid

\section{Introduction}

Lung cancer is the most commonly found malignant disease worldwide, and the leading cause of death due to cancer. About 80 percent of lung cancer patients are affected by tumors with non-small cell histologies (NSCLC); and $30-40 \%$ of them at diagnosis have locoregionally limited, but-for tumor extension or medical reasons-inoperable diseases in stages I-III.

For stage I patients increasingly a stereotactic approach is chosen, whereas for locoregionally advanced stages more fractionated forms of radiotherapy are the cornerstone of treatments, often combined with chemotherapy.

State of the art treatments for locoregionally advanced NSCLC comprise 60 Gy simultaneously applied to 2 cycles of chemotherapy. However, for toxicity reasons this approach is amenable only for about $30 \%$ of patients [1]. Furthermore, the premature closure of RTOG 0617 randomizing 60 Gy vs. 74 Gy suggests, that improvement of the results by intensifying radiation is improbable [2].

In contrast, sequential chemo-radiotherapeutic modalities seem to offer promising possibilities. A positive doseresponse relationship in the range 60-100 Gy has been established for tumor control and survival [3]; and there is growing evidence that shortening of the overall treatment time is crucial $[4,5]$. However, until now radiation doses in escalation trials are always determined by dose constraints for normal tissues, not by features of tumors relevant for tumor control $[3,6,7]$. As a consequence, larger tumors in these trials are often treated with smaller doses than small tumors.

Recently, in our clinic the conformal target splitting technique has been developed for treating lung cancer, attended by a concept of rather tight margins in the treatment planning process [8-11]. Initially, high dose radiation therapies with conventional fractionation rendered encouraging results [12]. Thereafter, in order to lower treatment times, in a phase I/II trial up to 90 Gy to primary tumors and 63 Gy to nodes in 1.8 Gy bid fractions were applied, preceded by 2 cycles chemotherapy [13]. The results showed good tolerability and promising results for tumor control and survival. The here presented consecutive trial targets directly the doses required for tumor control in a differentiated mode. Radiation doses were related to tumor volume, treating larger tumors with higher doses. Primary endpoints are the local and regional tumor control rates at 2 years (as $>90 \%$ of locoregional failures occur within 2 years). Secondary endpoints are survival and toxicity. With a minimum follow-up time of 2 years for patients alive, the final results of this prospective trial are presented.

\section{Methods and materials \\ Trial design}

Eligible were patients with nonresected, histologically/ cytologically proven NSCLC in stages I through III B, and a Karnofsky Index $=>60$.

Radiotherapy had to be given twice daily with fractional doses of 1.8 Gy (ICRU Report 50), an interval of $>10 \mathrm{~h}$, at 5 days/week. Overall doses to the primary tumors were aligned along increasing tumor size within 4 groups in the range 73.8-90.0 Gy (Tables 1 and 2). The dose to macroscopically involved nodes had to be 54.0-72.0 Gy (adapted to the degree of extent and invasion, left to the discretion of the treating physician), to elective nodes $45.0 \mathrm{~Gy}$ within a region of about $6 \mathrm{~cm}$ cranial to macroscopically involved nodes. In stage I patients elective nodal irradiation could have been omitted.

The number of cycles of induction chemotherapy was left to the discretion of the referring department; preferably it should have been limited to two cycles. The interval between chemotherapy and radiotherapy should have been < 8 days. Simultaneous chemotherapy was not allowed.

\section{Staging procedures}

Staging evaluations included a medical history, physical examination, chest X-ray, bronchoscopy, 18-fluorodeoxyglucose-positron emission tomography (FTG-PET) and a CT-scan or MRI of the brain.

\section{Radiotherapy planning and delivery}

Patients were set up in vacuum cradles, usually supine with the hands above the head. Planning CTs in treatment position were performed as 'slow CTs', with patients freely breathing (non-spiral CT; $4 \mathrm{~s}$ /slice; slice thickness $7 \mathrm{~mm}$ ) or in a few patients as 4D-CT/average projection at the end of the accrual period (internal target volume concept, grossly summarizing and depicting the different positions of the moving tumor, rendering 


\begin{tabular}{|c|c|}
\hline Age, years, median & $66(44-87)$ \\
\hline Gender: m/f, n & $119 / 41$ \\
\hline Weightloss > 5\%/3 month, n (\%) & $38(24)$ \\
\hline \multicolumn{2}{|l|}{ Karnofsky Index, n (\%) } \\
\hline 60 & $10(6)$ \\
\hline 70 & $72 / 45)$ \\
\hline $80-100$ & $78(49)$ \\
\hline \multicolumn{2}{|l|}{ Histology/cytology, n (\%) } \\
\hline Squamous cell carcinoma & $104(63)$ \\
\hline Adenocarcinoma & $43(26)$ \\
\hline NSC-n. o. S & $17(11)$ \\
\hline \multicolumn{2}{|l|}{ AJCC-stage, n (\%) } \\
\hline I & $38(24)$ \\
\hline$\|$ & $6(4)$ \\
\hline III A & $69(43)$ \\
\hline III B & $47(29)$ \\
\hline \multicolumn{2}{|l|}{ Affiliation according PT- $\varnothing, \mathrm{n}(\%)$} \\
\hline Group $1(<2.5 \mathrm{~cm})$ & $27(17)$ \\
\hline Group $2(2.5-4.5 \mathrm{~cm})$ & $94(57)$ \\
\hline Group $3(4.5-6.0 \mathrm{~cm})$ & $30(18)$ \\
\hline Group 4 (> $6.0 \mathrm{~cm})$ & $13(8)$ \\
\hline \multicolumn{2}{|c|}{ Gross tumor volume (ccm, median/mean, range) } \\
\hline Group 1 & 17/33 (2-167) \\
\hline Group 2 & $50 / 68(9-492)$ \\
\hline Group 3 & $92 / 108(50-205)$ \\
\hline Group 4 & $163 / 238(105-572$ \\
\hline \multicolumn{2}{|l|}{ Tumor localisation, n (\%) } \\
\hline Central & $44(27)$ \\
\hline Peripheral & $120(73)$ \\
\hline
\end{tabular}

Abbreviations: NSC-n.o.s. $=$ Non small cell-not otherwise specified; $\mathrm{AJCC}=$ American Joint Commission on Cancer, $\mathrm{PT}-\varnothing=$ Primary tumor diameter (mean number of 3 perpendicular diameters).

dispensable an extra-margin for tumor movement) [10]. A margin of $7 \mathrm{~mm}$ was added to the gross tumor volume (GTV) to draw the planning target volume (PTV). In general, pretherapeutic PET-CT scans were visually studied by the treating physician before drawing the PTV; only in cases of atelectasis a second, postchemotherapeutic PET$\mathrm{CT}$ was performed and PET- and planning-CT scans were digitally matched. In patients receiving chemotherapy, the PTV was delineated at the postchemotherapy scans. PTVs of primary tumors and lymph nodes were drawn within the lung window and soft tissue window, respectively.

For patients with locoregionally advanced disease mostly the conformal target splitting technique was used $[8,9]$.

Treatment plans were generated in a 'forward' planning process, minimizing especially radiation to healthy
Table 2 Treatment characteristics

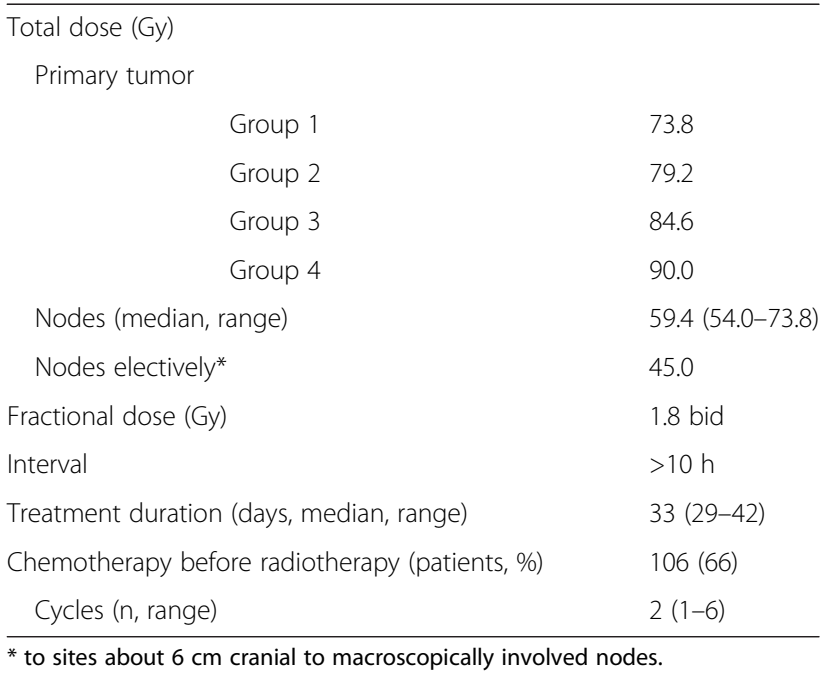

lung tissues, irrespective of its level or volume. In general the following lung dose constraints were applied: V20 (volume receiving $>20$ Gy) for a single lung $50 \%$, V25 for both lungs 30\%; in some patients these constraints were surpassed. A dose constraint for spinal cord was set at $45 \mathrm{~Gy}$; and for esophagus at 80 Gy (measured in the center of the esophagus at its most exposed level). Examples of treatment plans have been published recently [9].

Treatments were applied by $15 \mathrm{MV}$ photons.

The set-up of patients was adjusted in 3 dimensions before every treatment, matching anatomical structures as esophagus, trachea, main bronchi by means of two $\mathrm{kv}$-images in two dimensions [14].

\section{Medicinal agents}

Chemotherapeutically, in general cisplatin or carboplatin containing doublets were used.

If parts of the esophagus were within or near the PTV, an antimycotic prophylaxis was given (Amphotericine B lozengers, 4 times daily, during the full course of radiotherapy) $[15,16]$.

\section{Follow-up procedures}

Patients were seen for assessment of toxicity and tumor control 6 and 12 weeks after the end of radiotherapy, then every 3 months for the first year, every 4 months during the second and third year, hence every six months. At the first control a chest X-ray, at all other controls thoracic CT-scans were performed. Local or regional tumor progression was diagnosed, if there was an increase in tumor volume compared with the previous CT-scan. In case of doubt a FDG-PET had to be performed.

Acute and late toxicity was scored according to the RTOG/EORTC criteria except for pulmonary toxicity 
grade 1, because the criterion 'mild symptoms of dry cough or dyspnea on exertion' is common in these pulmonary compromised patients. As in our experience in rare cases pneumonitis as an acute side effect can be present until 6 months after therapy, toxicity is considered late if it persisted or developed beyond 6 months after the completion of radiotherapy.

\section{Statistical analysis}

Overall survival and local tumor control rates were calculated using the Kaplan-Meier method. All time intervals refer to the start of therapy, induction chemotherapy included. The median follow up time for all patients is 26.0 months (3.8-96), for survivors 38.1 months (24.896 months). No patient has been lost to follow-up.

In order to collect sufficient data in the four treatment groups, it was prospectively determined to enrol at least 150 patients in this trial.

The study was performed with consent of the medical ethics committee of the province Salzburg. All patients gave informed consent.

\section{Results}

\section{Patient and treatment parameters}

From January 2004 until December 2009160 patients with 164 histologically/cytologically proven NSCLC were enrolled. This corresponds to $96 \%$ of all NSCLC patients in stages I-IIIB referred to our department in this period (patients with malignant pleural effusions, pancoast tumors and one patient with pulmonal fibrosis excluded, see below). Five of the 7 patients not enrolled presented with $\mathrm{KI}<60 \%$, two patients refused to be treated twice daily for geographical reasons. Hence we consider the study population as continuously referred and unselected.

Patient and tumor characteristics are shown in Table 1. Notably, 24\% presented with $>5 \%$ weight loss during the three months preceding diagnosis; and $51 \%$ had a $\mathrm{KI}<80 \%$.

Treatment parameters can be seen in Table 2. All but five primary tumors received the dose corresponding to the respective treatment group (in 3 patients of group 2 and in 2 patients of group 3, doses were lowered for 3 fractions; for dose/volume issues or a reduced general status of the patients). The vast majority of stage II/III patients received an elective nodal treatment cranial to macroscopically involved sites. For dose-volume reasons in 18 patients $(15 \%)$ this elective treatment was not performed.

\section{Local and regional tumor control, distant failures}

We observed 32 local failures, 30 of them occurred within 2 years; this results in an actual 2- and actuarial 5 -year local tumor control rate of $77 \%$ and $74 \%$, respectively (Figure 1a). The distribution of local recurrences within the four groups can be seen in Figure 1b. The best results show the patients in group 1 (90\% tumor control at 2 years), the control rates in group 2 to 4 are similar and lie between $65 \%$ and $76 \% / 2$ years.

10 patients showed an isolated regional failure (without a local recurrence) at a median time of 14 months (range 8-26 months), corresponding to a regional control rate of $93 \%$ at 2 years (Figure 1c). Five of these failed at distant sites simultaneously. In 6 patients the regional failure was located in originally irradiated sites (54.0$63.0 \mathrm{~Gy}$ ), in 4 patients in non-treated regions (three of these four patients belong to the small group of 18 patients, where an elective nodal treatment primarily was omitted).

In 57 patients (36\%) hematogenous metastases appeared during the course of the disease, mostly in the brain (23 patients).

\section{Overall survival}

The actual overall 2 year-survival rate for all patients and the actuarial 5 year-survival rate is $57 \%$ and $19 \%$, respectively, the median survival time 28.0 months (Figure 2a). The overall median and 5 year-survival rate for patients in stage I, II, IIIA, IIIB, IIIA + B amounts to 43.4, 33.1, 22.0, $27.8,24.3$ months and to $22 \%, 33 \%, 22 \%, 11 \%, 18 \%$, respectively (Figures $2 \mathrm{~b})$. Survival curves according to tumor size groups are depicted in Figure 2c.

30 patients died from intercurrent diseases at a median time of 15.3 months (range 3.8-72.1 months). Nine of them have had stage I tumors and were referred to primary RT due to a low performance status.

\section{Toxicity}

Table 3 shows the non-hematologic toxicities. In two patients a treatment-related death occurred: progressive pulmonary fibrosis (not pneumonitis) 5 and 6 months after the end of radiotherapy. The patients were treated for stage IIIA tumors, with unexceptional pulmonary doses (V25 30\% and 23\%, respectively). In the pretherapeutic CT scans of both patients clear radiologic signs of existing pulmonary fibrosis were visible. These events occurred within 2 months during the second year of the accrual period. Thereafter patients with pulmonary fibrosis were excluded from enrolment in this trial. Apart from that, treatments were well tolerated.

One patient showed late esophageal toxicity grade 3 (stenosis, 7 months after the end of radiotherapy with moderate esophageal doses, treated by stenting; a few months later the patient died for a local recurrence). Almost all patients present slight to moderate posttherapeutic densities in the lung parenchyma, which did not cause symptoms $>$ grade 2 .

Four patients died from a pulmonary hemorrhage. In three of them a recurring central tumor was diagnosed 


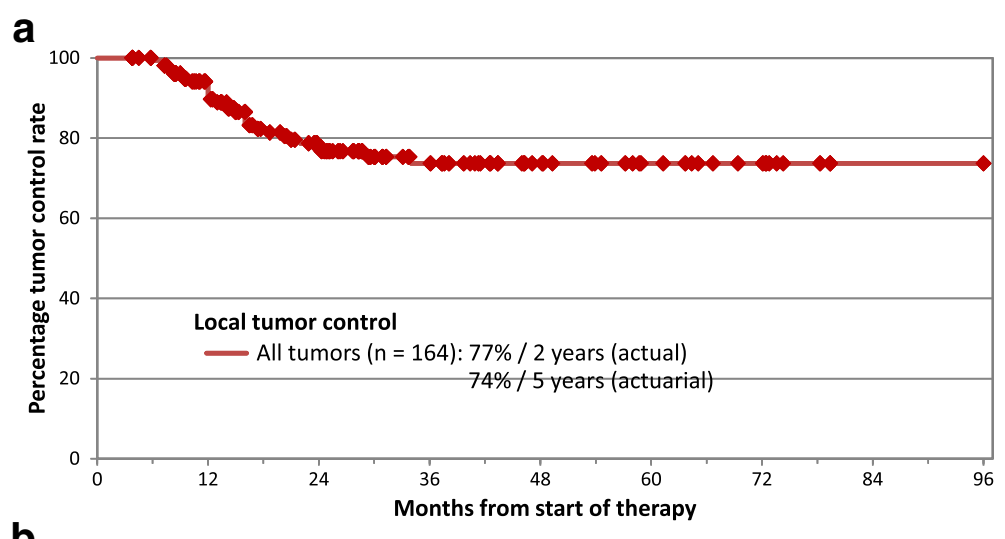

b

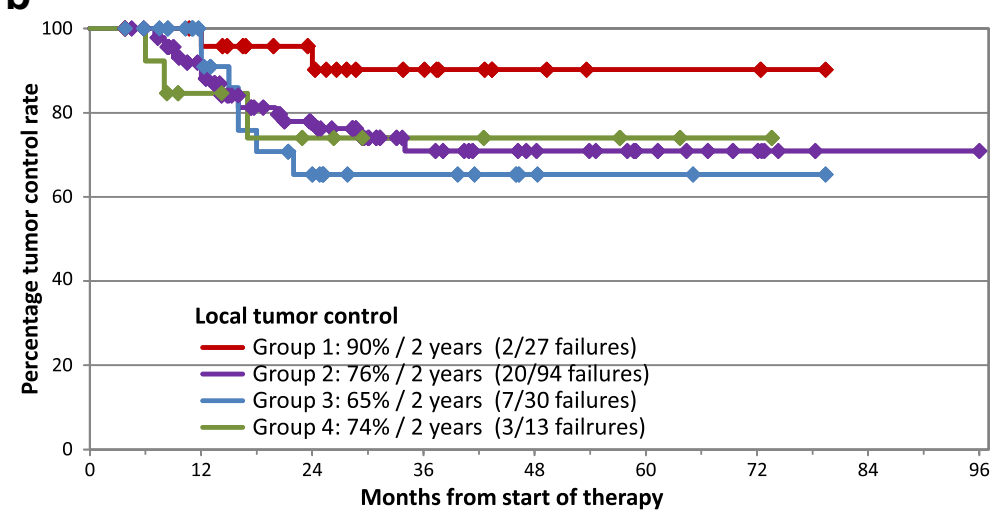

C

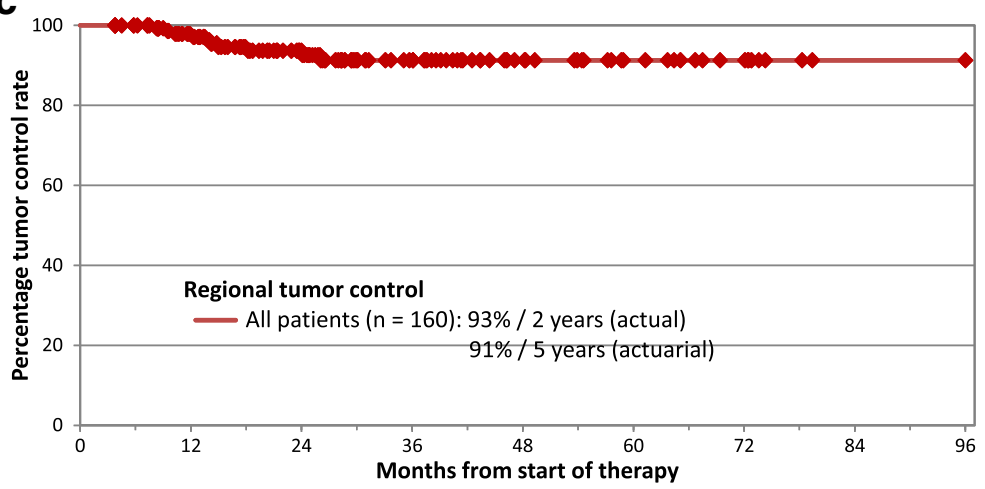

Figure 1 Kaplan-Maier plots for a) local tumor control all tumors; b) local tumor control by tumor size groups; c) regional tumor control all patients.

before. The fourth patient, treated with 79.2 Gy for a central tumor with close proximity to the bronchial arteries for about $4 \mathrm{~cm}$, died 6.5 months after finishing radiotherapy. The autopsy demonstrated a leakage of a bronchial artery without detection of a recurrent tumor, nor necrotic tissue. The most probable cause of death of this patient is a brochoarterial fistula arisen after retraction of the central, infiltrating tumor.

\section{Discussion}

We consider the concept and mode of implementation of DART-bid a novel approach for treating non-resected
NSCLC. Usually in dose escalation protocols, for reasons of tolerability, doses are determined by dose constraints for normal tissues, not by features relevant for tumor control as e.g. tumor size $[3,6,7,17-19]$. As a consequence large tumors are often treated with smaller doses than small tumors and presumably high volume tumors are occasionally excluded from enrolment in protocols. DART-bid targets straightforward the doses required for a high level of tumor control. New is also the differentiation of doses on tumor sites: it is sufficient to treat nodes with lower doses compared to primary tumors, because generally nodes are well oxygenized, without necrotic areas and of smaller size. 

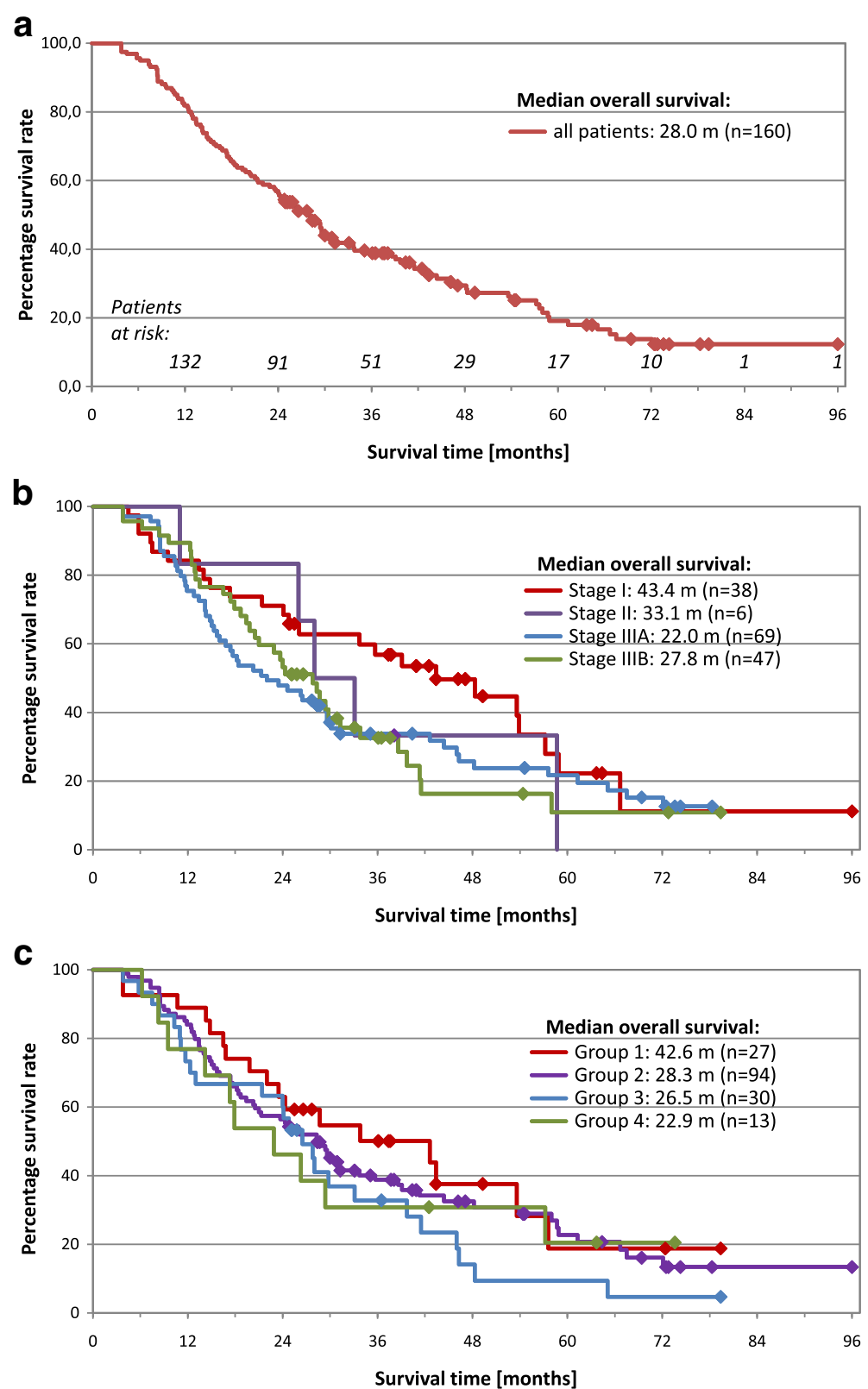

Figure 2 Overall survival. Kaplan-Maier plots for a) all patients; b) patients by tumor stage; c) patients by tumor size groups.

Table 3 Acute (A) and late (B) non-hematologic toxicity according to EORTC/RTOG criteria, $\mathbf{n}(\%)$

\begin{tabular}{lllllll}
\hline & & Grade 1 & Grade 2 & Grade 3 & Grade 4 & Grade 5 \\
\hline A & Esophagus & $51(32)$ & $16(10)$ & $7(4)$ & - & - \\
& Lung & NA & $10(7)$ & $6(4)$ & - & $2(1)$ \\
B & Esophagus & - & - & $1(1)$ & - & - \\
& Lung & NA & $15(9)$ & - & - & - \\
\hline
\end{tabular}

Abbreviations: EORTC/RTOG = European Organisation for Research and Treatment of Cancer/Radiation Therapy Oncology Group.; NA = not assessed.
Table 4 shows that in our series the results compare favourably with the outcome of other modalities in all endpoints: local and regional tumor control, survival, toxicity.

\section{Local tumor control}

In reported studies of simultaneous radio-/chemotherapies and dose escalation protocols locoregional tumor control is mostly not appropriately examined [1,3,6,20,21]. Corresponding diagnostic methods and intervals are not prospectively performed. Often only the patterns of first relapses are reported. Simultaneous radio-/chemotherapies 
Table 4 Selected trials in the treatment of NSCLC

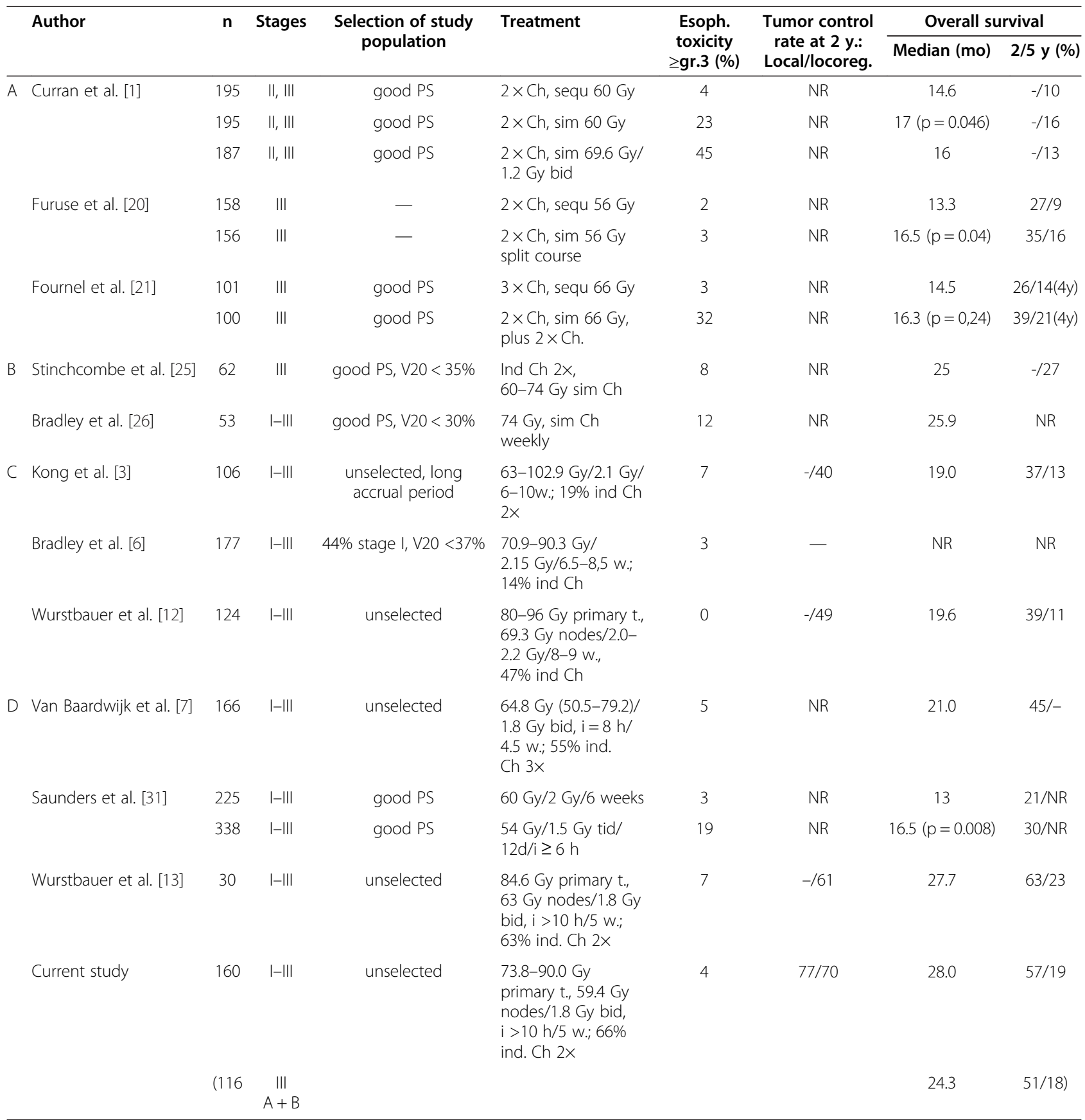

A. Randomized studies comparing sequential versus simultaneous radio-/chemotherapy. B. Further simultaneous studies. C. Radiation dose escalation trials. D. Trials employing accelerated radiotherapy.

Abbreviations: $\mathrm{NR}=$ not reported; $\mathrm{Ch}=$ chemotherapy; sequ $=$ sequential, $\operatorname{sim}=$ simultaneous, ind $=$ induction, $\mathrm{PS}=\mathrm{Performance}$ status.

seem to achieve definitive local tumor control in about $30-40 \%$ of the patients $[1,20,21]$, sequential high-dose treatments with conventional fractionation in about $40-$ $50 \%[12,22]$.

Total dose levels in our protocol were set with the intention of a high degree of tumor control, according to our experience in the preceding phase I/II trial [13]. A control rate of $90 \%$ for the small tumors (group 1) and between $65 \%$ and $76 \%$ for the larger ones (group 2-4) seems to be impressive, although there is still space for improvement. In our patients, image guidance was performed in every fraction, matching anatomical structures as trachea, main bronchi, esophagus via two orthogonal kv-images. Recently, in a series of 30 patients we compared the topographic accuracy matching anatomical structures as described versus matching on gold fiducials, 
implanted bronchoscopically in or near the primary tumor. In 8 out of 30 patients $(27 \%)$ in $>30 \%$ of fractions the vector of the position corrected via fiducials exceeded $7 \mathrm{~mm}$, which is our commonly used margin from GTV to PTV. In other words, without position correction along fiducials, in a quarter of the patients in more than a third of the fractions parts of the GTV come to lie outside the treated volume. Hence, a further improvement of local control might not primarily be a matter of raising the total dose, but of raising geographic accuracy. Therefore, in our successive series of NSCLC patients, tending to further improve local tumor control, total doses remain unchanged and IGRT with gold fiducials (or cone beam CT) is performed routinely.

It has been discussed, if local tumor control should be adjudged only if tumors initially respond with a partial or complete response radiologically (RECIST-criteria) [23]. We cannot share this opinion, because occasionally we observed lung tumors presenting at CT-controls as opacities in dimensions of a minor response for periods longer than 10 years.

\section{Regional tumor control}

The regional tumor control rate of $93 \%$ in our study is high and confirms the concept to apply lower doses to nodes in comparison to primary tumors.

Regarding elective nodal irradiation, in four patients an isolated nodal recurrence in previously untreated sites occurred. Only one of these patients belong to the group of 104 stage II/III patients treated primarily electively cranial to macroscopically involved nodes, whereas three belong to the small group of 18 patients, in which an elective nodal irradiation was postponed for volume issues. This confirms the necessity of elective nodal radiation cranial to involved nodes. In general nowadays, elective nodal irradiation has been abandoned, in order to gain potential to raise the dose to macroscopic tumor; but series conducted in this way report isolated elective nodal recurrences in up to $9 \%$ of the patients $[6,24]$.

\section{Survival}

A median overall survival time of 28.0 months for all patients and 24.3 months for stage III patients compares favourably with the results of other approaches (Table 4). Simultaneous chemo-/radiotherapies result in survival times of about 17 months [1,20,21] (Table 4). The patients in these studies were not PET staged and therefore an imbalance for stage migration may occur; however, in contrast to our unselected population, these patients were in good general condition, without considerable comorbidities and without weight loss. There are simultaneous treatments applying 74 Gy, achieving survival times up to 26 months; in these series the additional selection criterion of a relatively strict V20 of $30-35 \%$ is used, most probably a surrogate for patients with lower 'tumor load' [25,26].

\section{Toxicity}

Except the two patients with pre-existing pulmonary fibrosis (see above), treatments were tolerated very well. We attribute the good tolerability and the fact, that $97 \%$ of the primary tumors could have been treated with the dose of the respective group, to three reasons: the beam arrangements inherent to the target splitting technique, the use of rather tight margins and the differentiation of doses [8-10]. These issues have been discussed in detail in the report of the preceding phase I/II trial [13]. Briefly, target splitting is a technique with a high potential of sparing organs at risk. Concerning the tight margin of only $7 \mathrm{~mm}$ from GTV to PTV: the internal target volume concept, using slow planning CTs or average mode $4 \mathrm{D}$ planning CTs, averts the necessity of adding a general extra margin for tumor motion (internal margin). And, as in stereotactic radiotherapy, we consider a margin for microscopic spread from GTV to a clinical target volume (CTV) in high dose radiotherapy dispensable, because a sufficient dose to the rim of microscopic disease (about 45 Gy in 2,5 weeks) is delivered anyway.

\section{Simultaneous vs. sequential therapies with intensified radiation component}

At present simultaneous chemo-/radiotherapies are considered state of the art for locoregionally advanced NSCLC. Several randomized trials have proven an advantage of $60 \mathrm{~Gy}$ applied simultaneously vs. sequentially $[1,20,21]$. The radiosensitizing chemotherapeutic effect improves the local tumor control, median overall survival times raise from about 14 to 17 months. As also side effects act simultaneously, these therapies are more toxic; therefore only about $30 \%$ of stage III patients are amenable for this approach [1]. A further, grave drawback of the simultaneous approach is the lack of improvements raising radiation doses. RTOG 0617, randomizing 60 Gy vs. 74 Gy recently was closed prematurely. An interim analysis showed that 74 Gy could not produce a survival benefit and possibly give worse results [2]. Final analysis must be expected, but 60 Gy, applied with 3D-conformal and IMRT techniques, seem to be the upper dose limit in simultaneous radio/chemotherapies.

In contrast, sequential therapies with intensified radiation components may offer promising developments.

The potential of 2 cycles induction chemotherapy to eradicate peripheral micrometastases, thus lowering the incidence of later distant relapse, has been proven in trials $[27,28]$ (and often induction chemotherapies also provide an advantegous shrinkage of tumors before starting radiotherapy). However, as discussd in detail in 
our phase I/II trial report, fast repopulation of tumor cells between cycles of chemotherapy and during the interval between chemotherapy and radiotherapy might occur $[13,29,30]$.To prevent this, we avoid to apply more than 2 cycles chemotherapy, and try to keep the interval to radiotherapy $<8$ days.

Similarly, a short overall radiation treatment time is crucial [5]. Fowler estimates a loss of local tumor control of $11 \%$ per week for treatment times beyond 4 weeks [4].This reflects the drawback of dose escalation protocols performed with conventional fractionation $[3,6,12]$ (Table 4). One way to lower radiation treatment time is treating patients more often than once daily $[7,13,31]$ (Table 4). Van Baardwijk et al. report on 1.8 Gy bid treatments with an individualized prescription based on normal tissue dose constraints (19 Gy mean lung dose) [7]. A median dose of 64.8 Gy (range 50.4-79.2 Gy) was applied and a median overall survival time of 21.0 months was achieved. Equal doses to primary tumors and nodes were given. Treatments were safe, figures for locoregional tumor control are not reported. DART-bid enables the application of higher doses; and regarding the low toxicity, these doses could even be raised, if necessary. The latter is investigated in ongoing studies, in the mode described above.

Another method to lower treatment times is hypofractionation. Mature result of such regimes are not yet available. Substantial side effects, especially concerning central tumors, could be an issue in this setting.

\section{Conclusions}

DART-bid following induction chemotherapy yields a high level of locoregional tumor control and survival times. In general it is well tolerated. In all outcome parameters it seems to compare favourably with simultaneous chemoradiotherapies, while being additionally amenable for an unselected patient population.

\section{Competing interests}

The authors declare that they have no competing interests.

\section{Authors' contributions}

KW designed the study, collected the data and drafted the manuscript. HD developed the various specific techniques employed. KW, KD, PK, FZ, BL, PP, BW, MS and FS treated the patients. All authors read and approved the final manuscript.

\section{Author details}

'Department of Radiation Oncology and radART-Institute for research and development on Advanced Radiation Technologies, Paracelsus Medical University, Salzburg, Austria. ${ }^{2}$ Department of Pneumology, Paracelsus Medical University, Salzburg, Austria. ${ }^{3}$ Universitätsklinik für Radiotherapie, Müllner Hauptstrasse 48, A-5020 Salzburg, Austria.

Received: 8 December 2012 Accepted: 25 February 2013 Published: 5 March 2013

\section{References}

1. Curran WJ, Paulus R, Langer C, Komaki R, Lee JS, Hauser S, Movsas B, Wasserman T, Rosenthal SA, Gore E, Machtay M, Sause W, Cox JD: Sequential vs. concurrent chemoradiation for stage III non-small lung cancer: Randomized phase III trial RTOG 9410. J Natl Cancer Inst 2011, 103:1452-1460.

2. Cox JD: Are the results of RTOG 0617 mysterious? Int I Radiat Oncol Biol Phys 2012, 82:1042-1044

3. Kong FM, Randall K, Ten Haken RK, Schipper MJ, Sullivan MA, Chen M, Lopez C, Kalemkerian GP, Hayman JA: High-dose radiation improved local tumor control and overall survival in patients with inoperable/ unresectable non-small cell lung cancer: Long-term results of a radiation dose escalation study. Int J Radiat Oncol Biol Phys 2005, 63:324-333.

4. Fowler J: Biological factors influencing optimum fractionation in radiation therapy. Acta oncol 2001, 40:712-717.

5. Mauguen A, Le Péchoux C, Saunders M, Schild S, Turrisi A, Baumann M, Sause W, Ball D, Belanie C, Bonner J, Zajusz A, Dahlberg S, Nankivell M, Mandrekar S, Paulus R, Behrendt K, Koch R, Bishop J, Dische S, Arriagada R, De Ruysscher D, Pignon JP: Hyperfractionated or accelerated radiotherapy in lung cancer: An individual patient data meta-anlysis. J Clin Oncol 2012, 30:2788-2797.

6. Bradley J, Graham M, Winter K, Purdy JA, Komaki R, Roa WH, Ryu JK, Bosch W, Emami B: Toxicity and outcome results of RTOG 9311: A phase I-II dose escalation study using three-dimensional conformal radiotherapy in patients with inoperable non-small cell lung carcinoma. Int J Radiat Oncol Biol Phys 2005, 61:318-328.

7. Van Baardwijk A, Wanders S, Boersma L, Borger J, Öllers M, Dingemans A, Bootsma G, Geraedts W, Pitz C, Lunde R, Lambin B, De Ruysscher D: Mature results of an individualized radiation dose prescription study based on normal tissue constraints in stages I-III non-small cell lung cancer. J Clin Oncol 2010, 28:1380-1386.

8. Wurstbauer K, Deutschmann H, Kranzinger M, Merz F, Rahim H, Sedlmayer F, Kogelnik HD: Radiotherapy for lung cancer: Target splitting by asymmetric collimation enables reduction of radiation doses to normal tissues and dose escalation. Int J Radiat Oncol Biol Phys 1999, 44:333-341.

9. Wurstbauer K, Deutschmann H, Kopp P, Merz F, Schöller H, Sedlmayer F: Target splitting in radiation therapy for lung cancer: further developments and exemplary treatment plans. Radiat Oncol 2009, 4:30.

10. Wurstbauer K, Deutschmann H, Kopp P, Sedlmayer F: Radiotherapy planning for lung cancer: Slow CTs allow the drawing of tighter margins. Radiother Oncol 2005, 75:165-170.

11. Guckenberger M: Evidence for the use of modern irradiation techniques for non-small cell lung cancer: unnecessary, inadequate, insufficient or too late? Strahlenther Onkol 2012, 188:287-288.

12. Wurstbauer K, Weise H, Deutschmann H, Kopp P, Merz F, Studnicka M, Nairz O, Sedlmayer F: Non-small cell lung cancer in stages I-IIIB: Long-term results of definitive radiotherapy with doses $>80$ Gy in standard fractionation. Strahlenther Onkol 2010, 186:551-557.

13. Wurstbauer K, Deutschmann H, Kopp P, Kranzinger M, Merz F, Nairz O, Studnicka M, Sedlmayer F: Nonresected non-small cell lung cancer in stages I through IIIB: Accelerated, twice daily, high-dose radiotherapy-a prospective phase I/II trial with long-term follow-up. Int J Radiat Oncol Biol Phys 2010, 77:1345-1351.

14. Deutschmann H, Steininger P, Nairz O, Kopp P, Merz F, Wurstbauer K, Zehentmayr F, Fastner G, Kranzinger M, Kametriser G, Kopp M, Sedlmayer F: Augmented reality' in conventional simulation by projection of 3-D structures into 2-D images. Strahlenther Onkol 2008, 184:93-99.

15. Wurstbauer K, Merz F, Sedlmayer F: Prophylactic administration of Amphotericine B to prevent esophagitis in thoracic radiotherapy-a prospective study. Strahlenther Onkol 2009, 185:512-516.

16. De Ruysscher D, Van Meerbeck J, Vandecasteele K, Oberije C, Pijls M, Dingemans AM, Reymen B, Van Baardwijk A, Wanders R, Lammering G, Lambin P, De Neve W: Radiation-induced esophagitis in lung cancer patients: Is susceptibility for neutropenia a risk factor? Strahlenther Onkol 2012, 188:564-567.

17. Song C, Pyo H, Kim J, Lim YK, Kim WC, Kim HJ, Kim DW, Cho KH: Superiority of conventional intensity-modulated radiotherapy over helical tomotherapy in locally advanced non-small lung cancer. Strahlenther Onkol 2012, 188:901-909.

18. Govaert S, Troost E, Schuurbiers O, Geus-Oei L, Termeer A, Span P, Bussink J: Treatment outcome and toxicity of intensity-modulated (chemo) 
radiotherapy in stage III non-small cell lung cancer patients. Radiat Oncol 2012, 7:150

19. Wang D, Sun J, Zhu J, Li X, Zhen Y, Sui S: Functional dosimetric metrics for predicting radiation-induced lung injury in non-small cell lung cancer patients treated with chemoradiotherapy. Radiat Oncol 2012, 7:69.

20. Furuse K, Fukuoka M, Kawahara M, Nishikawa H, Takada Y, Kudoh S, Katagami N, Ariyosh Y: Phase III study of concurrent versus sequential thoracic radiation therapy in combination with mitomycin, vindesine, and cisplatin in unresectable Stage III non-small cell lung cancer. J Clin Oncol 1999, 17:2692-2699.

21. Fournel $P$, Robinet $G$, Thomas $P$, Souquet $P J$, Lèna $H$, Vergnenégre $A$, Delhoume JY, Le Treut J, Silvani JA, Dansin E, Bozonnat MC, Daurés JP, Mornex F, Peról M: Randomized phase III trial of sequential chemoradiotherapy compared with concurrent chemoradiotherapy in locally advanced non-small cell lung cancer: Groupe Lyon-Saint Etienne d'Oncologie Thoracique-Groupe Francais de Pneumo-Cancérologie NPC 95-01 study. J Clin Oncol 2005, 23:5910-5917.

22. Guckenberger M, Kavanagh A, Partridge M: Combinig advanced radiotherapy technologies to maximize safty and tumor control probability in stage III non-small cell lung cancer. Strahlenther Onkol 2012, 188:894-900.

23. Machtay M, Paulus R, Moughan J, Komaki R, Bradley J, Choy H, Albain K, Movsas B, Sause W, Curran W: Defining local-regional control and its importance in locally advanced non-small cell lung cancer. J Thorac Oncol 2012, 7:716-722.

24. Rosenzweig K, Sura S, Jackson A, Yorke E: Involved-field radiation therapy for inoperable non-small cell lung cancer. J Clin Oncol 2007, 25:5557-5561

25. Stinchcombe TE, Lee CB, Moore DT, Rivera MP, Halle J, Limentani S, Rosenman JG, Socinski MA: Long-term follow-up of a phase I/II trial of dose escalating three-dimensional conformal thoracic radiation therapy with induction and concurrent Carboplatin and Paclitaxel in unresctable stage IIIA/B non-small lung cancer. J Thorac Oncol 2008, 3:1279-1285.

26. Bradley JD, Bae K, Graham MV, Byhardt R, Govindan R, Fowler J, Purdy JA, Michalski JM, Gore E, Choy H: Primary analysis of the phase II component of a phase $1 / /$ intensification study using three-dimensional conformal radiation therapy and concurrent chemotherapy for patients with inoperabile non-small cell lung cancer: RTOG 0117. J Clin Oncol 2010, 28:2475-2480.

27. Dillman RO, Seagren SL, Propert KJ, Guerra J, Eaton WL, Perry MC, Carey RW, Frei EF, Green MR, Engl N: A randomized trial of induction chemotherapy plus high-dose radiation versus radiation alone in Stage III non-small ling cancer. New Engl J Med 1990, 323:940-945.

28. Sause WT, Kolesar P, Taylor S, Johnson D, Livingston R, Komaki R, Emami B, Curran W Jr, Byhardt R, Dar AR, Turrisi A 3rd: Final results of a phase III trial in regionally advanced unresectable non-small cell lung cancer. Chest 2000, 117:358-364.

29. El Sharouni SY, Kal HB, Battermann JJ: Accelerated regrowth of non-small cell lung tumours after induction chemotherapy. Br J Cancer 2003, 89:2184-2189.

30. Davis A, Tannock I: Repopulation of tumour cells between cycles of chemotherapy: a neglected factor. Lancet Oncol 2000, 1:86-93.

31. Saunders MI, Dische S, Barrett A, Harvey A, Griffiths G, Palmar M: Continuous hyperfractionated accelerated radiotherapy (CHART) versus conventional radiotherapy in non-small cell lung cancer: mature data from the randomised multicenter trial. Radiother Oncol 1999, 52:137-148.

doi:10.1186/1748-717X-8-49

Cite this article as: Wurstbauer et al.: DART-bid (Dose-differentiated accelerated radiation therapy, 1.8 Gy twice daily)-a novel approach for non-resected NSCLC: final results of a prospective study, correlating radiation dose to tumor volume. Radiation Oncology 2013 8:49.

\section{Submit your next manuscript to BioMed Central and take full advantage of:}

- Convenient online submission

- Thorough peer review

- No space constraints or color figure charges

- Immediate publication on acceptance

- Inclusion in PubMed, CAS, Scopus and Google Scholar

- Research which is freely available for redistribution

Submit your manuscript at www.biomedcentral.com/submit
C Biomed Central 